\title{
Inhibitory effects of ethyl pyruvate administration on human gastric cancer growth via regulation of the HMGB1-RAGE and Akt pathways in vitro and in vivo
}

\author{
JING ZHANG, JIN-SHUI ZHU, ZHOU ZHOU, WEI-XIONG CHEN and NI-WEI CHEN \\ Department of Gastroenterology, Shanghai Sixth People's Hospital Affiliated to \\ Shanghai Jiaotong University, Shanghai 200233, P.R. China
}

Received November 9, 2011; Accepted December 12, 2011

DOI: $10.3892 /$ or.2012.1623

\begin{abstract}
The high mobility group box-B1 (HMGB1)-receptor for advanced glycation end-products (RAGE) and the protein kinase $\mathrm{B}(\mathrm{Akt})$ pathways play a crucial role in tumorigenesis and development of many malignant tumors. Ethyl pyruvate (EP), a potent inhibitor of HMGB1 release, can exert antitumor effects on the growth of gastric cancer. Therefore, it is necessary to observe the effects of EP on gastric cancer growth in vitro and in vivo. Human gastric adenocarcinoma tissues of different grades $(\mathrm{N}=45)$ were collected. The expression of HMGB1 and RAGE was evaluated immunohistochemically in biopsy samples. After SGC-7901 gastric cancer cells were treated with EP, the expression of HMGB1, RAGE, Akt, phosphorylated Akt (p-Akt) and some transcription factors was identified and the effects of EP on cell proliferation, invasion, cell cycle distribution and apoptosis were assessed. A subcutaneous xenograft tumor model was established, validating the effects of EP on tumor growth in vivo. The expression of HMGB1 and RAGE was respectively observed in 73.3 and $68.9 \%$ of the gastric adenocarcinoma tissues. The frequency of positive expression increased with the ascending grade of the tumor malignancy. EP decreased the expression of HMGB1, RAGE, Akt, p-Akt, Ki-67 and matrix metallopeptidase-9 (MMP-9), and increased the expression of p53. Moreover, EP could inhibit tumor cell proliferation and invasion, induce cell cycle arrest and apoptosis and slow the growth of xenograft tumors. In conclusion, HMGB1 and RAGE were strongly expressed in gastric adenocarcinoma, and EP administration inhibited gastric cancer growth via regulation of the HMGB1-RAGE and Akt pathways. EP may play a critical role in the treatment of cancer in conjunction with other therapeutic agents.
\end{abstract}

Correspondence to: Professor Jin-Shui Zhu, Department of Gastroenterology, Sixth People's Hospital Affiliated to Shanghai Jiaotong University, 600 Yishan Road, Shanghai 200233, P.R. China E-mail: zhujs1803@hotmail.com

Key words: ethyl pyruvate, high mobility group box-B1, receptor for advanced glycation end-products, growth, gastric cancer

\section{Introduction}

Gastric cancer is one of the most common malignancies worldwide, with an estimated 934,000 cases reported globally in 2002, and is the second most common cause of death from cancer. The prognosis of gastric cancer is poor with an estimated relative five year survival rate of less than $20 \%(1,2)$. Though the therapeutic approaches for cancer such as surgery, hormone therapy, radio- and chemo-therapy have some efficacy, expensive medical costs are still the main problem faced by many patients in developing countries. Efforts to improve cancer care in the developing world will benefit from the identification of simple, inexpensive, and broadly applicable medical modalities based on emergent innovations in treatment, such as targeting mechanisms of signaling pathways (3).

Gastric cancer is also a genetic disease developing from a multi-step process (4). Single or multiple mutations in genes related to growth control, apoptosis, invasion and metastasis form the molecular genetic basis of malignant transformation and tumor progression (5). Mounting evidence argues that the high mobility group box-B1 (HMGB1)-RAGE and protein kinase $\mathrm{B}$ (Akt) pathways play a crucial role in the development and progression of many malignant tumors, and have been considered as important therapeutic targets. HMGB1, a chromatin-associated nuclear protein and extracellular damage-associated molecular pattern molecule, is an evolutionarily ancient and critical regulator of cell death and survival. Overexpression of HMGB1 is associated with each of the hallmarks of cancer including unlimited replicative potential, ability to develop angiogenesis, evasion of apoptosis, self-sufficiency in growth signals, insensitivity to inhibitors of growth and tissue invasion (6). HMGB1 is supposed to affect tumor growth by high affinity binding to RAGE (7). RAGE is the cellular receptor identified for extracellular HMGB1 and is associated with tumorigenesis and growth of gastric, esophageal, lung and colorectal cancer (8-11). Both HMGB1 and RAGE are expressed in hepatocellular, colorectal and breast cribriform carcinomas, and their expression is also associated with tumor development $(12,13)$. In addition, Akt is expressed in all cell types and tissues. Akt can contribute to cell survival and has become an effective antitumor target. Clinically, increased activity of Akt can lead to gastric cancer progression and proliferation, and is involved 
in cellular survival and proliferation signaling in human glioblastoma cells $(14,15)$. As such, targeting Akt signaling may be a potential therapeutic strategy $(16,17)$.

Pyruvate, a key metabolite in cellular energy metabolism, is the end-product of glycolysis and the starting substrate for the tricarboxylic acid cycle, which generates NADH and ATP synthesis during oxidative phosphorylation. Pyruvate has been shown in one study to display anti-inflammatory and antioxidant properties in vivo (18). Ethyl pyruvate (EP), a stable lipophilic pyruvate derivative, inhibits the release of TNF and HMGB1 from endotoxin-stimulated murine macrophages, as well as attenuates activation of both the p38 mitogen-activated protein kinase and NF- $\mathrm{KB}$ signaling pathways. EP treatment of septic mice decreases circulating levels of HMGB1. Pretreatment with EP also prevents endotoxin lethality, inhibits the release of TNF and HMGB1 and ameliorates murine colitis and renal ischemia and reperfusion injury by decreasing HMGB1 release (19-21). Delayed treatment with EP also suppresses tumor growth significantly, although to a lesser extent. Tumors have early, marked leukocytic infiltration, and EP administration decreases the innate and adaptive immune cell infiltrates in the liver. Serum IL-6 and HMGB1 levels, which are elevated following tumor injection, are decreased significantly in EP-treated animals (22). Moreover, necrosis-to-apoptosis switch activity of EP may contribute to its anti-inflammatory action and suppress tumor development, possibly through its activity to induce the cell death mode switch from tumor promoting necrotic cell death to tumor suppressive apoptotic cell death (23).

Therefore, as a low-cost and anti-inflammatory agent, the antitumor effects of EP need to be further explored. Currently, the molecular mechanisms of EP antitumor effects are mainly focused on inflammation and the immune response. However, the EP regulation of signaling pathways related to tumorigenesis is rarely reported. In this study, we examined the expression of HMGB1 and RAGE in gastric adenocarcinoma tissues and explored the effects and molecular mechanism of EP on gastric cancer growth in vitro and in vivo. We hypothesized that EP administration may suppress gastric cancer growth via regulation of the HMGB1-RAGE and Akt pathways.

\section{Materials and methods}

Materials. The SGC-7901 cell line used in the experiment was from the Institute of Biochemistry and Cell Biology (Shanghai, China); Six-week-old female immune-deficient nude mice (BALB/c-nu) were purchased from the Shanghai SLAC Laboratory Animal Co., Ltd. (Shanghai Laboratory Animal Center of Chinese Academy Sciences). The primers for HMGB1, RAGE, Akt and phosphorylated Akt (p-Akt) were synthesized by ABI Co., Ltd. (USA). All antibodies were from Santa Cruz Biotechnology, Inc. (Santa Cruz, CA, USA).

Drugs and reagents. Ethyl pyruvate was obtained from Sigma-Aldrich (St. Louis, MO, USA). 3-(4,5)-Dimethylthiazol(-2-yl)-2,5-diphenyltetrazolium bromide (MTT) was from Dingguo Biology (Shanghai, China); Dulbecco's modified Eagle's medium (DMEM) and fetal bovine serum (FBS) were from Thermo Fisher Scientific, Inc. (Waltham, MA, USA); TRIzol reagent and Lipofectamine 2000 were from
Invitrogen (Carlsbad, CA, USA); M-MLV reverse transcriptase was from Promega (Madison, WI, USA); the SYBR-Green Master Mixture was from Takara Bio, Inc. (Otsu, Japan); the cell cycle analysis kit and the apoptosis kit [propidium iodide (PI), RNase A, Annexin V-FITC] were from KeyGen Biology (Nanjing, China). the ECL-PLUS/kit was from GE Healthcare (Piscataway, NJ, USA).

Tissue samples. Forty-five freshly resected gastric adenocarcinoma samples were collected at the Department of Gastroenterology of the Shanghai Sixth People's Hospital during 2009 and were classified according to the 2000 World Health Organization classification. Tissues and clinical information were obtained as part of an approved study at Shanghai Jiaotong University. There were 10 cases of well-differentiated gastric adenocarcinoma tissues, 15 cases of moderatelydifferentiated gastric adenocarcinoma tissues, 20 cases of poorly-differentiated gastric adenocarcinoma tissues and 45 corresponding cases of adjacent non-cancer tissues. Ten cases of normal gastric tissue specimens were obtained from endoscopic patients with chronic gastritis. A portion of each tissue sample was fixed with $10 \%$ formalin for histopathological and immunohistochemical examination. All tumors and normal gastric tissues were diagnosed by two independent gastroenterologists. The expression of HMGB1 and RAGE in those samples was examined by immunohistochemistry. The correlation of immunohistochemical expression with the degree of gastric adenocarcinoma malignancy was determined.

Immunohistochemical staining. Formalin-fixed tissue samples were prepared as paraffin-embedded sections and stained with hematoxylin and eosin. Unstained sections were deparaffinized and incubated overnight at $4^{\circ} \mathrm{C}$ with primary antibodies against HMGB1 and RAGE and with biotinylated secondary antibody at room temperature for $1 \mathrm{~h}$, followed by incubation with $\mathrm{ABC}$ peroxidase and 3,3'-diaminobenzidine (DAB; $30 \mathrm{mg}$ dissolved in $100 \mathrm{ml}$ Tris-buffer containing $0.03 \% \mathrm{H}_{2} \mathrm{O}_{2}$ ). Sections were counterstained with hematoxylin. Expression of HMGB1 and RAGE in each specimen was scored according to the percentage of positive-stained cells present in five randomly selected high magnification fields: 0 , no expression; 1 , positive cell ratio $<25 \% ; 2$, positive cell ratio $26-50 \%$; and 3 , positive cell ratio $>50 \%$.

Cell culture. SGC-7901 cells were cultured in DMEM medium supplemented with $10 \%$ heat-inactivated FBS, $100 \mathrm{U} /$ $\mathrm{ml}$ of penicillin and $100 \mu \mathrm{g} / \mathrm{ml}$ of streptomycin. They were all placed in a humidified atmosphere containing $5 \% \mathrm{CO}_{2}$ at $37^{\circ} \mathrm{C}$.

Quantitative real-time PCR. To quantitatively determine the mRNA expression level, real-time PCR was used. Total-RNA of each clone was extracted with TRIzol according to the manufacturer's protocol. Reverse-transcription was carried out using M-MLV and cDNA amplification was carried out using SYBR-Green master mix kit according to the manufacturer's protocol. HMGB1, RAGE, Akt and p-Akt genes were amplified using specific oligonucleotide primers and the human glyceraldehyde-3-phosphate dehydrogenase (GAPDH) gene was used as an endogenous control. The PCR primer sequences were 
as follows: HMGB1, 5'-atatggcaaaagcggacaag-3' and 5'-aggccaggatgttctccttt-3'; RAGE, 5'-gtcatggaactgcccaaact-3' and 5'-tccttctgcggatctgtctt-3'; Akt, 5'-taggtgtggatggttgcaga-3' and 5'-ctcccacagacccagaacat-3'; p-Akt, 5'-cctggtgattgtggtgacag-3' and 5'-gggetggagaacgtagactg-3'; GAPDH, 5'-caacgaatttggctacagca-3' and 5'-aggggtctacatggcaactg-3'. Data were analyzed using the comparative $\mathrm{Ct}$ method $\left(2^{-\Delta \Delta \mathrm{Ct}}\right)$ (24). Three separate experiments were performed for each clone.

Western blot analysis. Cells of each group treated with different concentrations of EP $(0,10,20$ and $40 \mathrm{mmol} / \mathrm{l})$ were harvested and extracted using lysis buffer (Tris-HCl, SDS, mercaptoethanol, glycerol). Cell extracts were boiled for $5 \mathrm{~min}$ in loading buffer and then equal amount of cell extracts were separated on $15 \%$ SDS-PAGE gels. Separated protein bands were transferred into polyvinylidene fluoride (PVDF) membranes and the membranes were blocked in 5\% skim milk powder. The primary antibodies against HMGB1, RAGE, Akt, p-Akt, Ki-67, matrix metallopeptidase-9 (MMP-9) and p53 were diluted according to the manufacturer's instructions and incubated overnight at $4^{\circ} \mathrm{C}$. Then, horseradish peroxidase-linked secondary antibodies were added at a dilution ratio of 1:1,000, and incubated at room temperature for $2 \mathrm{~h}$. The membranes were washed with PBS for three times and the immunoreactive bands were visualized using the ECL-PLUS/kit according to the kit's instructions. The relative protein level in different cell lines was normalized to GAPDH concentration. Three separate experiments were performed for each clone.

Cell proliferation assay. Cell proliferation was analyzed with the MTT assay. Briefly, cells treated with different concentrations of EP were incubated in 96-well-plates at a density of $1 \times 10^{5}$ cells/well with DMEM medium supplemented with $10 \%$ FBS. Cells were treated with $20 \mu \mathrm{l}$ MTT dye at $0,24,48,72$, $96,120 \mathrm{~h}$ and then incubated with $150 \mu \mathrm{l}$ of DMSO for $5 \mathrm{~min}$. The color reaction was measured at $570 \mathrm{~nm}$ with an enzyme immunoassay analyzer (Bio-Rad, USA). The proliferation activity was calculated for each clone.

Colony formation assay. SGC-7901 cells treated with different concentrations of EP were counted and seeded in 12-well plates (in triplicate) at 100 cells/well. Fresh culture medium was replaced every three days. Colonies were counted only if they contained more than 50 cells, and the number of colonies was counted from the sixth day after seeding and then the cells were stained using crystal violet. The rate of colony formation was calculated with the equation: colony formation rate $=$ (number of colonies/number of seeded cells) x100\%.

Transwell invasion assay. Transwell filters were coated with matrigel $(3.9 \mu \mathrm{g} / \mu \mathrm{l}, 60-80 \mu \mathrm{l})$ on the upper surface of a polycarbonate membrane (diameter $6.5 \mathrm{~mm}$, pore size $8 \mu \mathrm{m}$ ). After incubating at $37^{\circ} \mathrm{C}$ for $30 \mathrm{~min}$, the matrigel solidified and served as the extracellular matrix for analysis of tumor cell invasion. Harvested cells $\left(1 \times 10^{5}\right)$ in $100 \mu$ of serum-free DMEM were added into the upper compartment of the chamber. A total of $200 \mu \mathrm{l}$ conditioned medium derived from NIH-3T3 cells was used as a source of the chemoattractant, and was placed in the bottom compartment of the chamber. After $24 \mathrm{~h}$ incubation at $37^{\circ} \mathrm{C}$ with $5 \% \mathrm{CO}_{2}$, the medium was removed from the upper chamber. The non-invaded cells on the upper side of the chamber were scraped off with a cotton swab. The cells that had migrated from the matrigel into the pores of the inserted filter were fixed with $100 \%$ methanol, stained with hematoxylin, and mounted and dried at $80^{\circ} \mathrm{C}$ for $30 \mathrm{~min}$. The number of cells invading through the matrigel was counted in three randomly selected visual fields from the central and peripheral portion of the filter using an inverted microscope (x200 magnification). Each assay was repeated three times.

Cell apoptosis analysis. To detect cell apoptosis, cells treated with different concentrations of EP were trypsinized, washed with cold PBS and resuspended in binding buffer according to the instructions of the apoptosis kit. FITC-Annexin V and PI were added to the fixed cells for $20 \mathrm{~min}$ in the dark at room temperature. Then, Annexin V binding buffer was added to the mixture before the fluorescence was measured on a FACSort flow cytometer. The cell apoptosis was analyzed using the CellQuest software (Becton-Dickinson, Mountain View, CA, USA). Three separate experiments were performed for each clone.

Cell cycle analysis. To detect cell cycle variation, cells treated with EP were trypsinized, washed with PBS and fixed with $80 \%$ cold ethanol overnight at $-20^{\circ} \mathrm{C}$. After PBS washing, the fixed cells were stained with PI in the presence of RNase A for $30 \mathrm{~min}$ at room temperature in the dark. Each sample was filtered through a $50 \mu \mathrm{m}$ nylon filter to obtain a single-cell suspension. The samples were then analyzed on a FACSort flow cytometer (Becton-Dickinson). ModFit 3.0 software (Verity Software House, Topsham, ME, USA) was used for cell cycle analysis. Three separate experiments were performed for each clone.

In vivo tumor xenograft studies. Four mice were injected subcutaneously with $1 \times 10^{8}$ SGC-7901 cells in $50 \mu 1$ of PBS pre-mixed with an equal volume of matrigel matrix (BectonDickinson). Mice were monitored daily, and three out of four mice developed a subcutaneous tumor. When the tumor size reached approximately $5 \mathrm{~mm}$ in length, they were surgically removed, cut into $1-2 \mathrm{~mm}^{3}$ pieces, and re-seeded individually into 21 other mice. When the tumor size reached approximately $5 \mathrm{~mm}$ in length, mice in the treatment groups were randomized and injected i.p. with EP $40 \mathrm{mg} / \mathrm{kg}(\mathrm{n}=7)$ and $80 \mathrm{mg} / \mathrm{kg}$ $(n=7)$. Control mice $(n=7)$ were injected with the same amount of PBS with the same schedule. Injections were repeated every day after initial treatment for 2 weeks. The tumor volume was measured every 3 days with a caliper, using the formula volume $=\left(\right.$ length $\mathrm{x}$ width $\left.{ }^{2}\right) / 2$.

Statistical analysis. The results of each experiment are presented as mean \pm SD where applicable. Statistically significant difference in each assay were determined by SPSS version 11.5. Difference in each group was tested for significance using the Kruskal-Wallis $\mathrm{H}$ test and ANOVA analysis of variance. A P-value $<0.05$ was considered significant.

\section{Results}

Expression of HMGBl and RAGE in gastric adenocarcinoma. Expression of HMGB1 and RAGE was respectively observed 


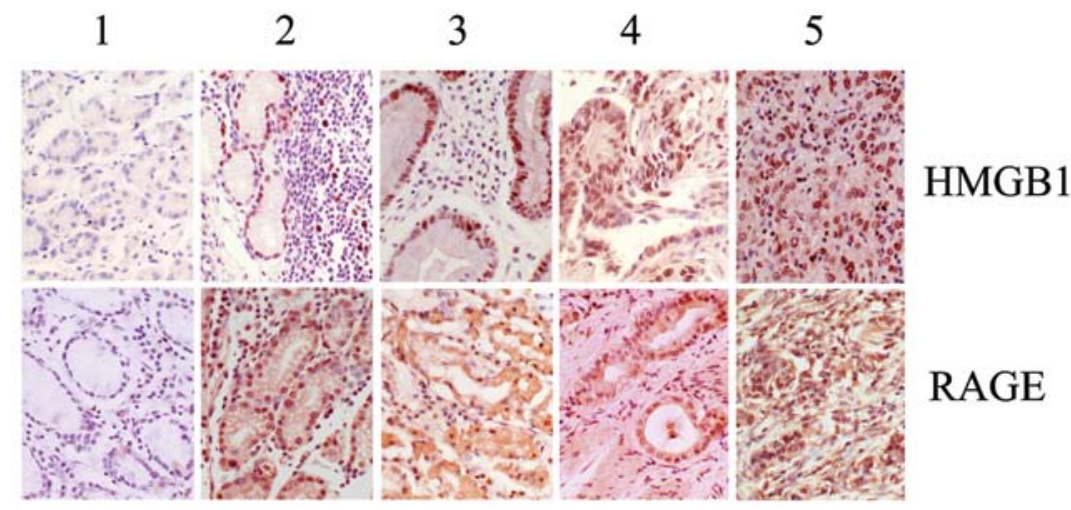

Figure 1. Expression of HMGB1 and RAGE in different grades of gastric adenocarcinoma (x200). HMGB1 and RAGE were expressed in gastric adenocarcinomas tissues, and frequency of positive expression increased with the ascending grade of the tumor malignancy. 1, Normal gastric mucous tissue; 2 , adjacent non-cancer tissue; 3, well-differentiated gastric adenocarcinoma tissue; 4, moderately-differentiated gastric adenocarcinoma tissue; 5, poorly-differentiated gastric adenocarcinoma tissue.

Table I. Expression of HMGB1 and RAGE in different grades of gastric adenocarcinoma tissues.

\begin{tabular}{|c|c|c|c|c|c|c|c|c|c|}
\hline \multirow[b]{2}{*}{ Target } & \multirow[b]{2}{*}{ Grade } & \multicolumn{4}{|c|}{$\mathrm{N}$} & \multirow[b]{2}{*}{ Total } & \multirow[b]{2}{*}{ Positive rate $(\%)$} & \multirow[b]{2}{*}{$\chi^{2}$} & \multirow[b]{2}{*}{$\mathrm{P}$-value } \\
\hline & & + & ++ & +++ & ++++ & & & & \\
\hline \multirow[t]{6}{*}{ HMGB1 } & $\mathrm{a}$ & 7 & 3 & 0 & 0 & 10 & 30.0 & & \\
\hline & $\mathrm{b}$ & 21 & 12 & 7 & 5 & 45 & 53.3 & 9.24 & 0.010 \\
\hline & $\mathrm{c}$ & 12 & 15 & 10 & 8 & 45 & 73.3 & & \\
\hline & $\mathrm{d}$ & 5 & 4 & 1 & 0 & 10 & 50.0 & & \\
\hline & $\mathrm{e}$ & 3 & 7 & 3 & 2 & 15 & 80.0 & 7.06 & 0.029 \\
\hline & $\mathrm{f}$ & 4 & 4 & 6 & 6 & 20 & 80.0 & & \\
\hline \multirow[t]{6}{*}{ RAGE } & $\mathrm{a}$ & 8 & 2 & 0 & 0 & 10 & 20.0 & & \\
\hline & $\mathrm{b}$ & 25 & 9 & 7 & 4 & 45 & 44.4 & 10.10 & 0.006 \\
\hline & $\mathrm{c}$ & 14 & 15 & 9 & 7 & 45 & 68.9 & & \\
\hline & $\mathrm{d}$ & 6 & 3 & 1 & 0 & 10 & 40.0 & & \\
\hline & $\mathrm{e}$ & 4 & 6 & 3 & 2 & 15 & 73.3 & 6.66 & 0.036 \\
\hline & $\mathrm{f}$ & 4 & 6 & 5 & 5 & 20 & 80.0 & & \\
\hline
\end{tabular}

a, normal gastric mucous tissue; b, adjacent non-cancer tissue; c, gastric adenocarcinoma tissue; d, well-differentiated gastric adenocarcinoma tissue; e, moderately-differentiated gastric adenocarcinoma tissue; f, poorly-differentiated gastric adenocarcinoma tissue.

in 73.3 and $68.9 \%$ gastric adenocarcinoma tissues (Fig. 1). The expression levels of HMGB1 and RAGE were elevated with ascending grade of tumor malignancy (Table I). Spearman rank correlation analysis showed a positive correlation between HMGB1 and RAGE expression in gastric adenocarcinoma.

Effects of EP administration on the expression of HMGB1$R A G E$ and the Akt pathway in gastric cancer cells. It has been proven that the HMGB1-RAGE and Akt pathways are highly expressed in SGC-7901 cells in preliminary experiments $(9,25,26)$. To examine the effects of EP administration on HMGB1, RAGE, Akt and p-Akt expression in gastric cancer cells, SGC-7901 cells were treated with different concentrations of EP. Quantitative real-time PCR was performed at $48 \mathrm{~h}$ recovery to measure their mRNA expression levels. The mRNA expression levels of HMGB1, RAGE, Akt and p-Akt were lower in the EP-treated groups compared to the control group. This decrease was dose-dependent (Fig. 2A). The EP inhibition of their protein expression was examined by western blot analysis (Fig. 2B). The protein levels of HMGB1, RAGE, Akt and p-Akt were dose-dependently decreased in EP-treated groups compared with the control group. Consequently, the HMGB1-RAGE and the Akt pathways in gastric cancer cells can be suppressed by EP administration.

Effects of EP administration on gastric cancer cell proliferation and independent growth. Deregulated cell proliferation is a hallmark of cancer (27). In order to examine the effects of EP administration on gastric cancer cell proliferation and independent growth, we investigated the proliferation and independent growth of SGC-7901 cells by MTT and colony formation assays. As a result, it was found that EP could significantly reduce the proliferative activity of SGC-7901 cells in a dose- and time-dependent manner in comparison with the control group (Fig. 3A). The colony formation rate of SGC-7901 cells was markedly lower in the EP-treated groups 


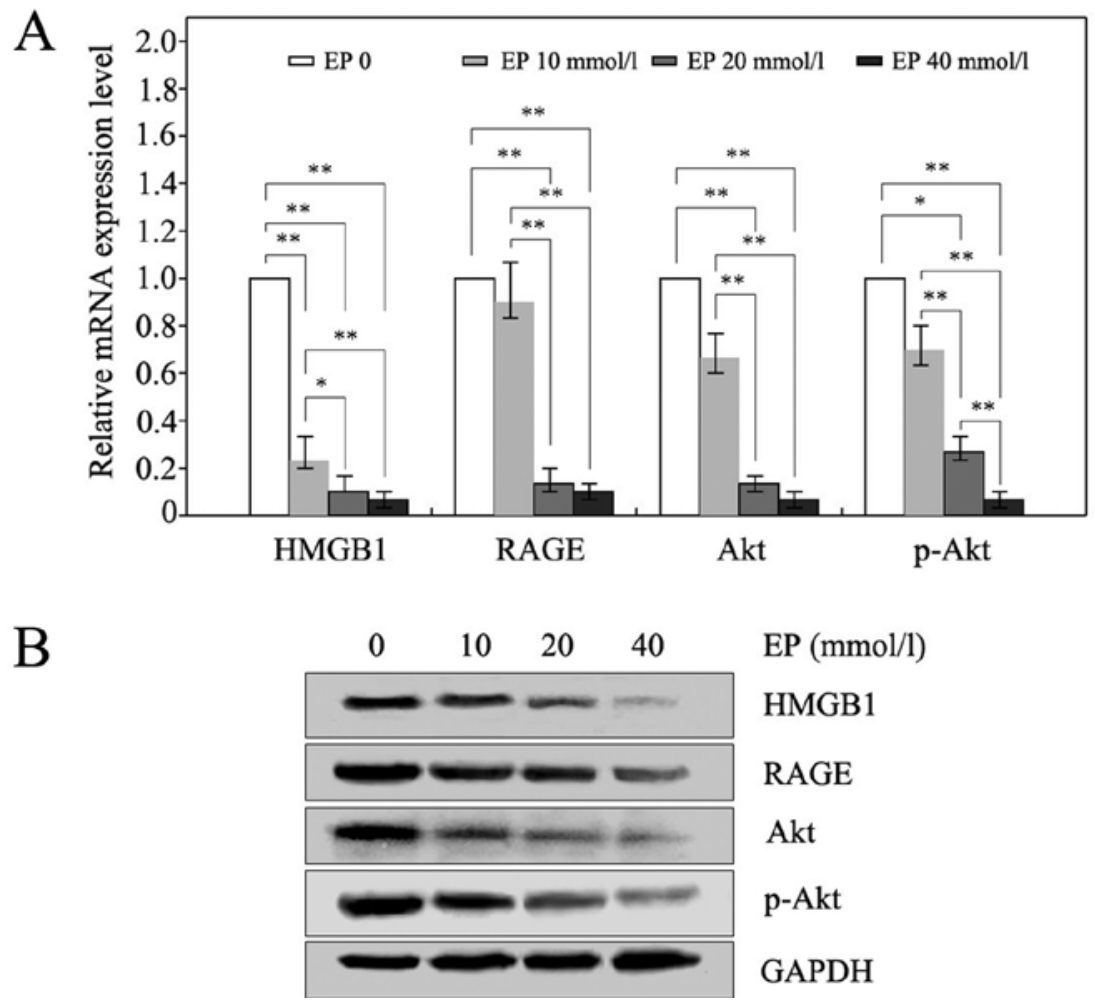

Figure 2. Suppression of HMGB1, RAGE, Akt and p-Akt expression in gastric cancer cells by EP administration. (A and B) The expression levels of HMGB1, RAGE, Akt and p-Akt were measured in SGC-7901 cells treated with different concentrations of EP by real-time PCR and western blotting, which showed that their expression levels were decreased by EP in a dose-dependent manner $\left({ }^{*} \mathrm{P}<0.05 ;{ }^{* *} \mathrm{P}<0.01\right)$

A

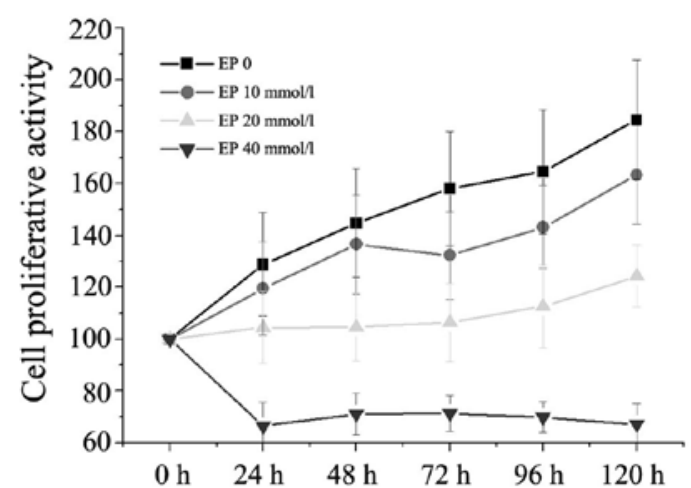

B
EP $0 \quad$ EP $10 \mathrm{mmol} / 1 \quad$ EP $20 \mathrm{mmol} / 1 \quad \mathrm{EP} 40 \mathrm{mmol} / \mathrm{l}$

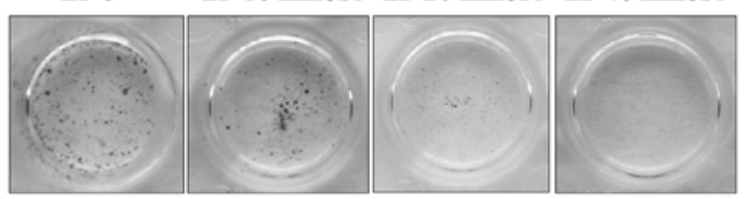

$\mathrm{C}$

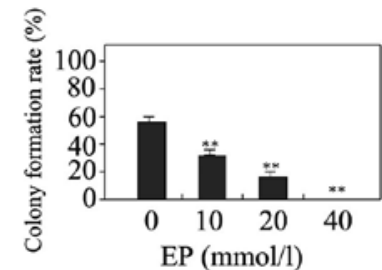

$\mathrm{D}$

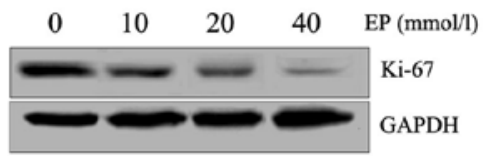

Figure 3. Suppression of gastric cancer cell proliferation and independent growth by EP administration. (A) SGC-7901 cells were treated with gradually increasing concentrations of EP from 0 to $40 \mathrm{mmol} / \mathrm{l}$ and the dose-dependent anti-proliferative effect was detected by the MTT assay. (B and C) Cell independent growth activity was detected by the colony formation assay. SGC-7901 cells were treated with gradually increasing concentrations of EP, and then seeded in 12 -well plates. The number of colonies was counted from the sixth day after seeding and the colony formation rate was calculated and shown ( $\left.{ }^{* *} \mathrm{P}<0.01\right)$. (D) The Ki-67 protein level was detected by western blot analysis. GAPDH protein was used as an endogenous control.

than in the control group (Fig. 3B and C). Ki-67 is at the very heart of many essential cellular processes and determines the tumor progression and the outcome of anticancer treatment. To determine whether EP suppressed endogenous Ki-67 through translational repression, the expression of $\mathrm{Ki}-67$ protein was examined by western blotting. It was shown that Ki-67 protein levels were lower in the EP-treated groups compared with the control group (Fig. 3D), suggesting that EP administration inhibits cell proliferation and independent growth of gastric cancer cells via negative regulation of $\mathrm{Ki}-67$ protein expression. 


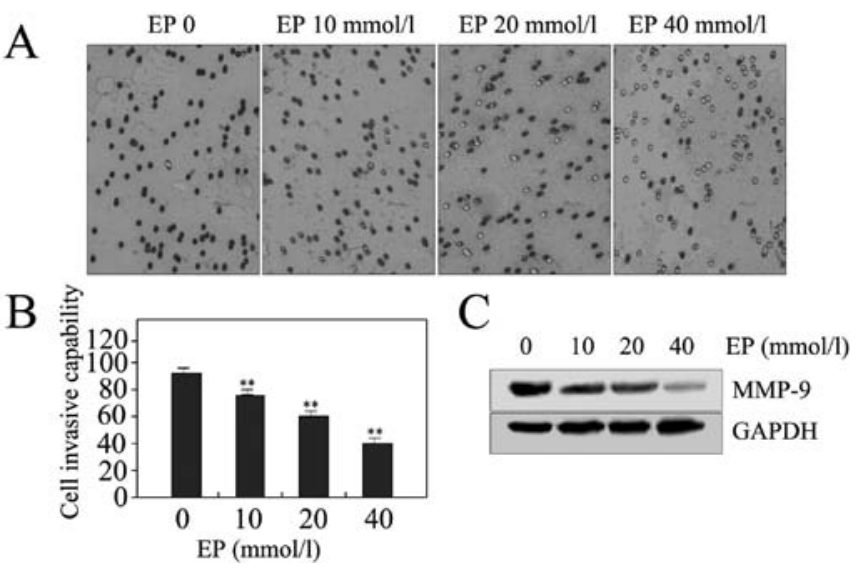

Figure 4. Suppression of gastric cancer cell invasion by EP administration. (A and B) The Transwell assay was performed to determine cell invasive capability. The number of SGC-7901 cells treated with gradually increasing concentrations of EP invading through the matrigel was significantly smaller than that of untreated cells $\left({ }^{* *} \mathrm{P}<0.01\right)$. (C) The expression level of MMP-9 protein was detected by western blot analysis. GAPDH protein was used as an endogenous control.

Effects of EP administration on gastric cancer cell invasion. To determine the effects of EP on gastric cancer cell invasion, a Transwell assay was performed. The results indicated that the invasive potential in the Transwell assay was determined on the basis of the ability of cells to invade a matrix barrier containing mainly laminin and type IV collagen, the major components of the basement membrane. Representative micrographs of Transwell filters can be seen in Fig. 4A. The invasive activities of SGC-7901 cells were distinctly decreased in the EP-treated groups compared with the control group (Fig. 4B). In terms of the important role of MMP-9 in tumor invasion, western blotting was performed to investigate the effects of EP on the expression of the MMP-9 protein. The protein level MMP-9 was significantly reduced in the EP-treated groups compared with the control group, indicating that EP inhibits gastric cancer cell invasion via negative regulation of MMP-9 protein expression (Fig. 4C).

Effects of EP administration on gastric cancer cell apoptosis and cycle distribution. To determine whether EP affected gastric cancer cell apoptosis and cycle distribution, flow cytometric analysis with PI/FITC-Annexin V staining was performed. The results showed that the apoptosis index of SGC-7901 cells in the EP-treated groups was markedly higher than in the control group (Fig. 5A and C). The cycle distribution of SGC-7901 cells was also analyzed and cell cycle kinetics showed that the $G_{0} / G_{1}$ phase fraction was increased, the $S$-phase fraction was decreased and cell cycle was arrested in the $G_{0} / G_{1}$ phase in the EP-treated groups (Fig. 5B). Additionally, the p53 pathway initiates DNA repair, cell-cycle arrest, senescence and, importantly, apoptosis (28). To determine whether EP affected the translational level of p53 protein, western blot was carried out. It was found that the protein level of p53 was increased in the EP-treated groups in comparison with the control group (Fig. 5D), suggesting that EP administration can induce gastric cancer cell apoptosis and block cell cycle progression through positive regulation of $\mathrm{p} 53$ protein expression.

Effects of EP administration on xenograft tumor growth. Our in vitro experiments demonstrated that $\mathrm{EP}$ administration could
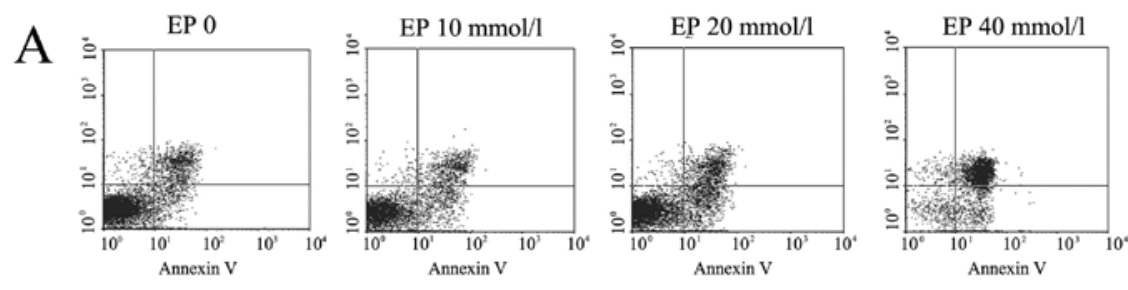

B
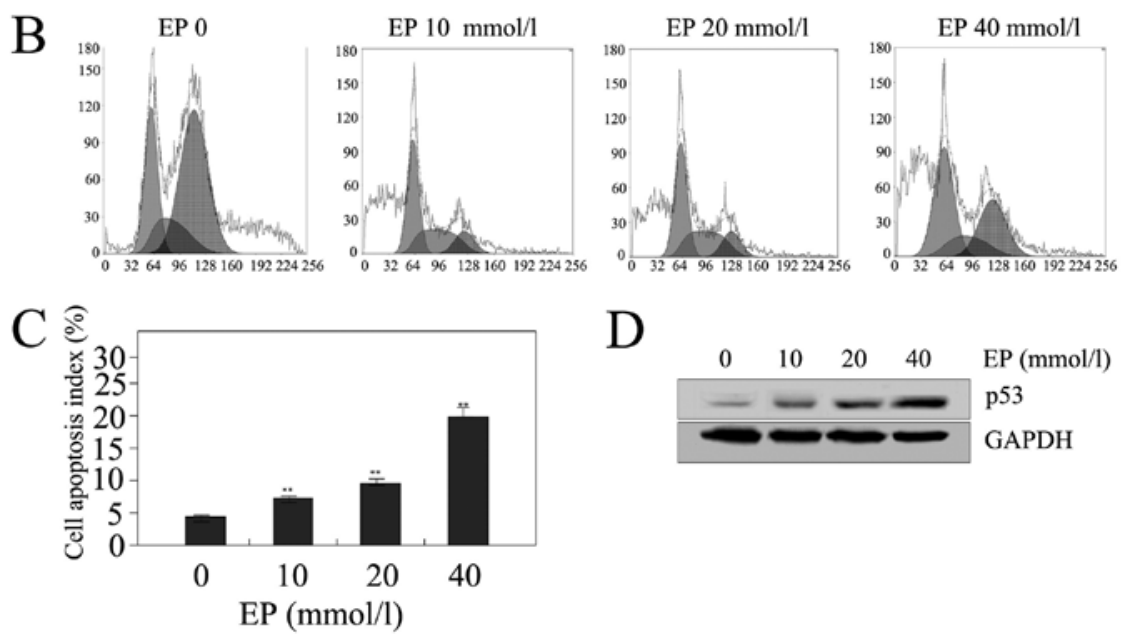

$\mathrm{D}$

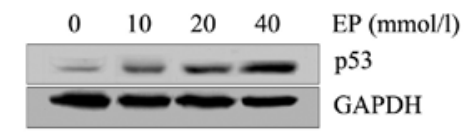

Figure 5. Induction of gastric cancer cell apoptosis and cell cycle arrest by EP administration. (A and C) Flow cytometric analysis with PI/FITC-Annexin V staining was performed to determine cell apoptosis. The apoptotic index of SGC-7901 cells treated with gradually increasing concentrations of EP was markedly higher than that of untreated cells $\left({ }^{* *} \mathrm{P}<0.01\right)$. (B) Cell cycle distribution was analyzed by flow cytometry, which showed that in EP-treated cells, the $\mathrm{G}_{0} /$ $\mathrm{G}_{1}$ phase fraction increased, the $\mathrm{S}$-phase fraction decreased and the cell cycle was arrested in the $\mathrm{G}_{0} / \mathrm{G}_{1}$ phase. (D) The expression level of teh p53 protein was detected by western blot analysis. GAPDH protein was used as an endogenous control. 

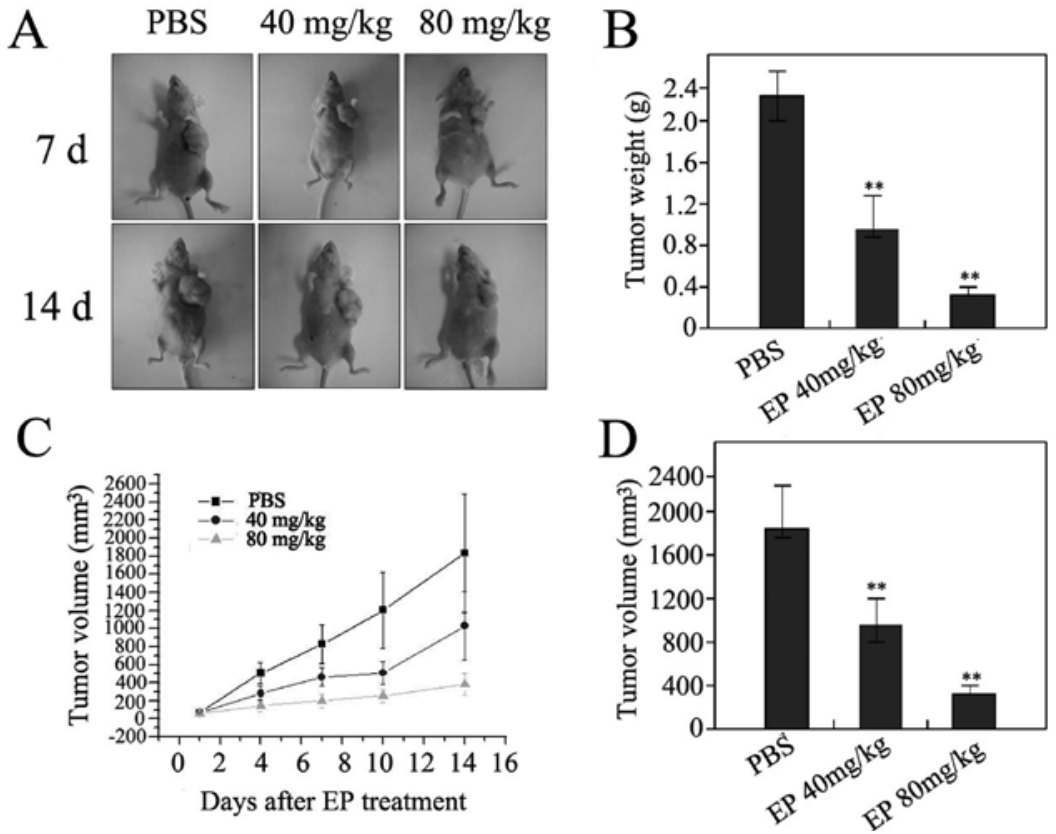

Figure 6. Suppression of the growth of xenograft tumor derived from SGC-7901 cells by EP administration. (A) The graph shows the difference of tumor volume between the EP-treated groups and the control group on Days 7 and 14. (C) Tumor volume was measured after EP treatment and the curve of the tumor volume is presented. (B and D) The differences of tumor weight and volume between the EP-treated groups and the control group were statistically significant $\left({ }^{*} \mathrm{P}<0.05 ;{ }^{* *} \mathrm{P}<0.01\right)$.

efficiently inhibit the growth of gastric cancer cells. Therefore, it is necessary to further investigate the effects of EP on xenograft tumor growth in vivo. The mean volume of tumors in all experimental mice before treatment was $67.01 \pm 14.38 \mathrm{~mm}^{3}$. During the whole tumor growth period (Fig. 6A and C), the tumor growth activity was measured. Tumors treated with 40 or $80 \mathrm{mg} / \mathrm{kg}$ EP grew substantially more slowly compared with the control group. When the tumors were harvested, the average weight and volume of the tumors in the EP-treated groups were significantly smaller than those of the control group (Fig. 6B and D). Also, there was a difference in tumor weight and volume between the EP 40 and $80 \mathrm{mg} / \mathrm{kg}$ groups. This result was consistent with the effects of EP in vitro and strongly suggested that EP administration could inhibit gastric cancer growth.

\section{Discussion}

The HMGB1-RAGE and the Akt pathways are closely associated with tumorigenesis, expansion and invasion of many cancers. Recent studies have shown that HMGB1 is critical for the survival of prostate cancer cells and targeted knockdown of HMGB1 mRNA can be used as a strategy to kill prostate cancer cells (29). HMGB1 also plays an important role in human lung cancer and downregulation of HMGB1 by RNA interference can inhibit invasion and proliferation of human lung cancer cells (30). RAGE, known as a multiligand receptor for certain stress-associated factors, has been considered to affect the characteristic differences of various cancer cells. Cromolyn binds S100P, prevents activation of RAGE, inhibits tumor growth and increases the effectiveness of gemcitabine in experimental models (31). MAbGB3.1 administration markedly reduces chronic inflammation and tumorigenesis in the mouse model of colitis-associated cancer and RAGE-deficient mice are resistant to the onset of colitis-associated cancer (32). Therefore, blockade of HMGB1-RAGE signaling pathways through some strategies could result in attenuation of tumor development and growth (33). In addition, aberrant loss or gain of Akt activation underlies the pathophysiological properties of tumor growth, proliferation and migration (34). Akt and p-Akt have recently been shown to be highly expressed in gastric carcinomas, and their expression may help predict the clinical outcome of gastric cancer patients (35). In a previous study, we confirmed that Akt1 is overexpressed in gastric cancer and targeted knockdown of Akt1 by RNA interference inhibits gastric cancer growth (26). HMGB1 and RAGE in this study were also found to be overexpressed in gastric adenocarcinoma tissues. Thus, some therapeutic strategies targeting the HMGB1-RAGE and Akt pathways could been adopted and applied to the treatment of cancer (36).

Interestingly, HMGB1 also functions as a tumor suppressor and radiosensitizer in breast cancer. HMGB1 is a nuclear protein containing a consensus RB (retinoblastoma)-binding LXCXE motif, and HMGB1-RB interaction is critical for the HMGB 1-mediated transcriptional repression, $\mathrm{G}_{1}$ cell cycle arrest, apoptosis induction and tumor growth suppression. Therefore, it is possible to design new therapies for the treatment of breast cancer that exert their effects by modulating the HMGB1 and RB regulatory pathway (37). However, it was found in our study that EP could inhibit the expression of HMGB1, RAGE, Akt and p-Akt in gastric cancer cells, and could further suppress tumor cell growth and induce apoptosis, suggesting that EP regulation of HMGB1-RAGE and Akt pathways may play some role in promoting tumorigenesis of gastric cancer. In fact, some studies have displayed EP regulation of cell signaling pathways. EP inhibits the $N F-\kappa B$ pathway through inhibition of the nuclear translocation of RelA in cultured lung epithelial cells and pVHL-mediated 
hypoxia inducible factor-1 (HIF-1) degradation via the MAPK pathway in cancer cells $(38,39)$. In addition, the role of inflammation in promoting tumor growth is becoming increasingly evident $(40,41)$. EP, as an anti-inflammatory agent, inhibits the growth of colorectal tumors in the liver, which is mediated by tumor (induction of apoptosis) and host (decreased inflammation) effects (22). It was also found that in our study EP could inhibit gastric cancer growth via regulation of the HMGB1RAGE and Akt pathways.

Moreover, the molecular mechanism of EP on gastric cancer growth in this study need be further explored. It is known that $\mathrm{Ki}-67$ is a nuclear protein that is expressed in proliferating cells and may be required for maintaining cell proliferation and is used as a marker for cell proliferation of gastric cancer (42). MMP-9 is thought to be a key enzyme involved in the degradation of type IV collagen. High levels of MMP-9 in tissues has been associated with tumor growth and invasion (43). p53 is one of the most mutated tumor suppressors in human cancers and participates directly in the intrinsic apoptosis pathway by interacting with the multidomain members of the Bcl-2 family to induce mitochondrial outer membrane permeabilization (44). It is reported that HMGB1 interferes with the apoptotic machinery at the level of caspase- 9 activation and inhibits cell apoptosis via c-IAP2 induction in the pathogenesis of colon carcinoma, while RAGE limits apoptosis through a p53-dependent mitochondrial pathway in tumor cell $(45,46)$. In our previous study, blockade of the PI3K/Akt pathway by small hairpin RNA inhibited the growth and metastasis of malignant tumors via negative regulation of Ki-67, MMP-9 and positive regulation of the tissue-inhibitor of metalloproteinase-2 (TIMP-2) and p53 (47). In this study, it was shown that EP suppressed gastric cancer growth via negative regulation of Ki-67, MMP-9 and positive regulation of p53, which might be mediated by the HMGB1-RAGE and Akt pathways.

Therefore, it is necessary to discuss the regulatory correlation between HMGB1 and Akt pathway. HMGB induces VEGF production in macrophage-like cells via activation of p38MAPK, ERK1/2 and Akt $(48,49)$. However, the PI3K/ Akt and the NF- $\mathrm{BB}$-related pathways in non-small cell lung cancer lead to the HMGB1-induced MMP-9 expression and cellular metastatic ability (50). In our study EP, as a HMGB1 inhibitor, could inhibit the expression of HMGB1 and Akt in gastric cancer cells, and it could be inferred that HMGB 1 might contribute to the Akt-induced MMP-9 expression and participate in tumor invasion.

In conclusion, EP displayed marked antitumor properties and inhibited gastric cancer growth via regulation of the HMGB1-RAGE and Akt pathways. More importantly, EP has been evaluated in phase II trials of high-risk cardiac patients undergoing cardiopulmonary bypass and has been shown to be safe and well-tolerated (51). Although there was no benefit conferred to these patients, EP and its potent analogs, such as methyl-2-acetamidoacrylate, have never been tested in the cancer setting in humans, an area that appears promising for clinical testing.

\section{Acknowledgements}

This study was supported by the Science and Technology Commission Animal Experiment Foundation of Shanghai,
China (grant no. 10140902500). We thank the members of the Chinese Academy of Sciences (Shanghai) for providing their technical assistance.

\section{References}

1. Parkin DM, Bray F, Ferlay J, et al: Global cancer statistics, 2002. CA Cancer J Clin 55: 74-108, 2005.

2. Neugut AI, Hayek M and Howe G: Epidemiology of gastric cancer. Semin Oncol 23: 281-291, 1996.

3. Iiizumi M, Liu W, Pai SK, et al: Drug development against metastasis-related genes and their pathways: a rationale for cancer therapy. Biochim Biophys Acta 1786: 87-104, 2008.

4. Kountouras J, Zavos C and Chatzopoulos D: New concepts of molecular biology on gastric carcinogenesis. Hepatogastroenterology 52: 1305-1312, 2005.

5. Tajima Y, Yamazaki K, Makino R, et al: Gastric and intestinal phenoty pic marker expression in early differentiated-type tumors of the stomach: clinicopathologic significance and genetic background. Clin Cancer Res 12: 6469-6479, 2006.

6. Tang D, Kang R, Zeh HJ III, et al: High-mobility group box 1 and cancer. Biochim Biophys Acta 1799: 131-140, 2010.

7. Taguchi A, Blood DC, del Toro G, et al: Blockade of RAGEamphoterin signalling suppresses tumour growth and metastases. Nature 405: 354-360, 2000.

8. Huttunen HJ and Rauvala H: Amphoterin as an extracellular regulator of cell motility: from discovery to disease. J Intern Med 255: 351-366, 2004.

9. Kuniyasu H, Oue N, Wakikawa A, et al: Expression of receptors for advanced glycation end-products (RAGE) is closely associated with the invasive and metastatic activity of gastric cancer. J Pathol 196: 163-170, 2002.

10. Jing RR, Cui M, Sun BL, et al: Tissue-specific expression profiling of receptor for advanced glycation end products and its soluble forms in esophageal and lung cancer. Genet Test Mol Biomarkers 14: 355-361, 2010.

11. Sasahira T, Akama Y, Fujii K, et al: Expression of receptor for advanced glycation end products and HMGB1/amphoterin in colorectal adenomas. Virchows Arch 446: 411-415, 2005.

12. Kostova N, Zlateva S, Ugrinova I, et al: The expression of HMGB1 protein and its receptor RAGE in human malignant tumors. Mol Cell Biochem 337: 251-258, 2010.

13. Ishiguro H, Nakaigawa N, Miyoshi Y, et al: RAGE and its ligand amphoterin are over expressed and associated with prostate cancer development. Prostate 64: 92-100, 2005.

14. Han Z, Wu K, Shen H, et al: Akt1/protein kinase B alpha is involved in gastric cancer progression and cell proliferation. Dig Dis Sci 53: 1801-1810, 2008.

15. Schlegel J, Piontek G, Budde B, et al: The Akt/protein kinase B-dependent anti-apoptotic pathway and the mitogen-activated protein kinase cascade are alternatively activated in human glioblastoma multiforme. Cancer Lett 158: 103-108, 2000.

16. Liu XH, Yu EZ, Li YY, et al: RNA interference targeting Akt promotes apoptosis in hypoxia-exposed human neuroblastoma cells. Brain Res 1070: 24-30, 2006.

17. Nakamura JL, Karlsson A, Arvold ND, et al: PKB/Akt mediates radiosensitization by the signaling inhibitor LY294002 in human malignant gliomas. J Neurooncol 71: 215-222, 2005.

18. Gupta SK, Rastogi S, Prakash J, et al: Anti-inflammatory activity of sodium pyruvate-a physiological antioxidant. Indian J Physiol Pharmacol 44: 101-104, 2000.

19. Ulloa L, Ochani M, Yang H, et al: Ethyl pyruvate prevents lethality in mice with established lethal sepsis and systemic inflammation. Proc Natl Acad Sci USA 99: 12351-12356, 2002.

20. Davé SH, Tilstra JS, Matsuoka K, et al: Ethyl pyruvate decreases HMGB1 release and ameliorates murine colitis. J Leukoc Biol 86: 633-643, 2009.

21. Chung KY, Park JJ and Kim YS: The role of high-mobility group box-1 in renal ischemia and reperfusion injury and the effect of ethyl pyruvate. Transplant Proc 40: 2136-2138, 2008.

22. Liang X, Chavez AR, Schapiro NE, et al: Ethyl pyruvate administration inhibits hepatic tumor growth. J Leukoc Biol 86: 599-607, 2009.

23. Lim SC, Choi JE, Kim CH, et al: Ethyl pyruvate induces necrosis-to-apoptosis switch and inhibits high mobility group box protein 1 release in A549 lung adenocarcinoma cells. Int J Mol Med 20: 187-192, 2007. 
24. Livak KJ and Schmittgen TD: Analysis of relative gene expression data using real-time quantitative PCR and the 2(-Delta Delta C(T)) method. Methods 25: 402-408, 2001.

25. Akaike H, Kono K, Sugai $\mathrm{H}$, et al: Expression of high mobility group box chromosomal protein-1 (HMGB-1) in gastric cancer. Anticancer Res 27: 449-457, 2007.

26. Zhang J, Zhang QY, Fu YC, et al: Expression of p-Akt and COX-2 in gastric adenocarcinomas and adenovirus mediated Aktl and COX-2 ShRNA suppresses SGC-7901 gastric adenocarcinoma and U251 glioma cell growth in vitro and in vivo. Technol Cancer Res Treat 8: 467-478, 2009.

27. Hanahan D and Weinberg RA: The hallmarks of cancer: the next generation. Cell 144: 646-674, 2011.

28. Vazquez A, Bond EE, Levine AJ, et al: The genetics of the p53 pathway, apoptosis and cancer therapy. Nat Rev Drug Discov 7: 979-987, 2008.

29. Gnanasekar M, Thirugnanam S and Ramaswamy K: Short hairpin RNA (shRNA) constructs targeting high mobility group box-1 (HMGB1) expression leads to inhibition of prostate cancer cell survival and apoptosis. Int J Oncol 34: 425-431, 2009.

30. Zhang P, Liu Y, Wu ZH, et al: Effects of high mobility group box-1 silencing upon the invasion and proliferation in human lung cancer cell L9981 by RNA inhibition. Zhonghua Yi Xue Za Zhi 89: 3156-3159, 2009 (In Chinese).

31. Arumugam T, Ramachandran V and Logsdon CD: Effect of cromolyn on S100P interactions with RAGE and pancreatic cancer growth and invasion in mouse models. J Natl Cancer Inst 98: 1806-1818, 2006

32. Turovskaya O, Foell D, Sinha P, et al: RAGE carboxylated glycans and S100A8/A9 play essential roles in colitis-associated carcinogenesis. Carcinogenesis 29: 2035-2043, 2008.

33. Hanford LE, Enghild JJ, Valnickova Z, et al: Purification and characterization of mouse soluble receptor for advanced glycation end products (sRAGE). J Biol Chem 279: 50019-50024, 2004.

34. Manning BD and Cantley LC: Akt/PKB signaling: navigating downstream. Cell 129: 1261-1274, 2007.

35. Nam SY, Lee HS, Jung GA, et al: Akt/PKB activation in gastric carcinomas correlates with clinicopathologic variables and prognosis. APMIS 111: 1105-1113, 2003

36. Han Z, Hong L, Wu K, et al: Reversal of multidrug resistance of gastric cancer cells by downregulation of Akt1 with Aktl siRNA. J Exp Clin Cancer Res 25: 601-606, 2006.

37. Jiao Y, Wang HC and Fan SJ: Growth suppression and radiosensitivity increase by HMGB1 in breast cancer. Acta Pharmacol Sin 28: 957-967, 2007.

38. Mizutani A, Maeda N, Toku S, et al: Inhibition by ethyl pyruvate of the nuclear translocation of nuclear factor- $\kappa \mathrm{B}$ in cultured lung epithelial cells. Pulm Pharmacol Ther 23: 308-315, 2010.
39. Kim SY, Choi JS, Park C, et al: Ethyl pyruvate stabilizes hypoxiainducible factor 1 alpha via stimulation of the TCA cycle. Cancer Lett 295: 236-241, 2010.

40. Vakkila $\mathbf{J}$ and Lotze MT: Inflammation and necrosis promote tumour growth. Nat Rev Immunol 4: 641-648, 2004

41. Kim S, Takahashi H, Lin WW, et al: Carcinoma-produced factors activate myeloid cells through TLR2 to stimulate metastasis. Nature 457: 102-106, 2009.

42. Czyzewska J, Guzińska-Ustymowicz K, Lebelt A, et al: Evaluation of proliferating markers Ki-67, PCNA in gastric cancers. Rocz Akad Med Bialymst 49 (Suppl 1): 64-66, 2004.

43. Cawston TE and Wilson AJ: Understanding the role of tissue degrading enzymes and their inhibitors in development and disease. Best Pract Res Clin Rheumatol 20: 983-1002, 2006.

44. Vaseva AV and Moll UM: The mitochondrial p53 pathway. Biochim Biophys Acta 1787: 414-420, 2009.

45. Völp K, Brezniceanu ML, Bösser S, et al: Increased expression of high mobility group box 1 (HMGB1) is associated with an elevated level of the antiapoptotic c-IAP2 protein in human colon carcinomas. Gut 55: 234-242, 2006.

46. Kang R, Tang D, Schapiro NE, et al: The receptor for advanced glycation end products (RAGE) sustains autophagy and limits apoptosis, promoting pancreatic tumor cell survival. Cell Death Differ 17: 666-676, 2010

47. Fu Y, Zhang Q, Kang C, et al: Inhibitory effects of adenovirus mediated COX-2, Akt1 and PIK3R1 shRNA on the growth of malignant tumor cells in vitro and in vivo. Int J Oncol 35: 583-591, 2009.

48. Park JS, Arcaroli J, Yum HK, et al: Activation of gene expression in human neutrophils by high mobility group box 1 protein. Am J Physiol Cell Physiol 284: C870-C879, 2003.

49. Kawahara K, Hashiguchi T, Kikuchi K, et al: Induction of high mobility group box 1 release from serotonin-stimulated human umbilical vein endothelial cells. Int J Mol Med 22: 639-644, 2008.

50. Liu PL, Tsai JR, Hwang JJ, et al: High-mobility group box 1-mediated matrix metalloproteinase- 9 expression in non-small cell lung cancer contributes to tumor cell invasiveness. Am J Respir Cell Mol Biol 43: 530-538, 2010.

51. Bennett-Guerrero E, Swaminathan M, Grigore AM, et al: A phase II multicenter double-blind placebo-controlled study of ethyl pyruvate in high-risk patients undergoing cardiac surgery with cardiopulmonary bypass. J Cardiothorac Vasc Anesth 23: 324-329, 2009 